EPJ Web of Conferences 113,05019 (2016)

DOI: $10.1051 /$ epjconf/201611305019

(C) Owned by the authors, published by EDP Sciences, 2016

\title{
Towards the next QCD Frontier with the Electron Ion Collider
}

\author{
Abhay Deshpande ${ }^{1, a}$, Zein-Eddine Meziani ${ }^{2, b}$, and Jian-Wei Qiu ${ }^{3,4, c}$ \\ ${ }^{1}$ Department of Physics and Astronomy, Stony Brook University, Stony Brook, NY 11794, USA \\ ${ }^{2}$ Department of Physics, Temple University, Philadelphia, PA 19122, USA \\ ${ }^{3}$ Physics Department, Brookhaven National Laboratory, Upton, NY 11973, USA \\ ${ }^{4}$ C.N. Yang Institute for Theoretical Physics and Department of Physics and Astronomy, Stony Brook Univer- \\ sity, Stony Brook, NY 11794, USA
}

\begin{abstract}
In this talk, we argue that the proposed Electron-Ion Collider (EIC) with its unique capability to collide polarized electrons with polarized protons and light ions at unprecedented luminosity, and with heavy nuclei at high energy, will be the most powerful tomographic scanner able to precisely image gluons and quarks inside the proton and nuclei. This precision microscope will allow us to "see" and explore the dynamics binding gluons and quarks together to form hadrons. The EIC will address the most compelling unanswered questions in QCD and hadron physics.
\end{abstract}

\section{Introduction}

Understanding the evolution of our universe from its origin (the Big Bang) to its current state, and the fundamental structure of all matters within it, is one of the most central goals of human scientific endeavor. This evolution is intimately tied to the properties of fundamental particles that existed in each of its phases as well the interactions that govern their dynamics. The discovery of the atomic structure and the nucleus by the Rutherford experiment over 100 years ago led to the discovery of quantum mechanics and the quantum world, which forever changed our view of our universe. It was the modern "Rutherford" experiment, namely the scattering between electrons and protons, performed at the Stanford Linear Accelerator Center (SLAC) in the sixties, that revealed quarks, the fundamental constituents of the proton, and led to the discovery of Quantum Chromo-Dynamics (QCD) - the theory of strong interaction between color charges that is responsible for confining colored quarks into color neutral hadrons through the exchange of gluons. In contrast to quantum electromagnetism where the force carriers ( photons ) are electrically neutral and non self-interacting, the force carriers (gluons) in QCD themselves carry a color charge causing them to interact among themselves. It is the non-linear gluon self-interaction that is responsible for both the confinement of color and the asymptotic freedom of the color force, two remarkable yet seemingly contradicting properties of QCD which make the strong interaction and hadron physics very rich, with plenty of dynamics mysteries.

\footnotetext{
ae-mail: abhay.deshpande@ stonybrook.edu

be-mail: meziani@temple.edu

ce-mail: jqiu@bnl.gov,Speaker
} 
Despite the great experimental challenge of probing the internal structure of a hadron without being able to observe the quarks and gluons in isolation, QCD has been extremely successful in interpreting data from all high energy $e^{+} e^{-}$, lepton-hadron and hadron-hadron collisions with momentum transfer(s) $\gg \Lambda_{\mathrm{QCD}} \sim 1 / \mathrm{fm}$. This was only possible due to the great theoretical development of QCD factorization formalisms which allows to connect the observed hadrons to the active quarks and gluons in high energy scattering. At the same time, the ab initio lattice QCD calculations have made tremendous progress and provided an impressive description of the mass spectrum of all known hadrons [1]. Today no one questions the validity of QCD as the fundamental theory of strong interactions in the Standard Model, and yet, our understanding of QCD remains incomplete. We know very little about the hadrons' quark-gluon structure and their emergence from quarks and gluons, a result closely connected to the defining property of QCD namely the color confinement.

The proposed Electron-Ion Collider (EIC) [2] will have highly polarized electrons, light ions and unpolarized nuclei (all the way to Uranium) colliding in the CoM energy range of 40-140 GeV, with a luminosity (e-nucleon equivalent) of $L_{e-N}=10^{33-34} \mathrm{~cm}^{-2} \mathrm{sec}^{-1}$. The EIC with such parameters will be able to address many overarching questions about QCD and hadron physics, such as:

- How are the sea quarks and gluons distributed in space and momentum inside the nucleon, and how do they build up nucleon's intrinsic properties, such as the spin?

- Does the gluon density saturate to produce the matter of universal properties in all hadrons, and is there a simple boundary that separates the saturated from more diluted quark-gluon matter?

- How does the nuclear environment affect the distribution of quarks and gluons and their interactions in nuclei, and how do hadrons emerge from quarks and gluons?

\section{The hadron structure}

The relation between the observable physical states, i.e. hadrons and the basic degrees of freedom, quarks and gluons (collectively called partons), is most intriguing in QCD. Proton structure is dynamic, not static, rich in features beyond our current knowledge and intuition. Quarks and gluons interact strongly when they are far apart - the confinement of color - but weakly when they are closer - the asymptotic freedom; they appear and disappear almost continuously; and they are confined to form the proton. Complete understanding of the proton structure and its properties (such as its mass, spin, size, etc...), the emergent phenomena of QCD dynamics, is still beyond the capability of today's best minds.

\subsection{Nucleon spin}

Several decades of experiments of deep inelastic scattering (DIS) of electron or muon beams off nucleons have taught us how partons share the momentum of a fast-moving nucleon. They have not, however, completely resolved the question of how partons build up the nucleon's spin? Following an intensive and worldwide experimental program over the past two decades, including recent measurements at RHIC, along with state-of-the-art QCD global analyses, we learned that the spin of quarks and antiquarks is only responsible for $\sim 30 \%$ of the proton spin, while the contribution from the gluons' spin is about $20-30 \%$ from the $x$-range explored so far. EIC will greatly increase the kinematic coverage in $x$ and $Q^{2}$, as shown in Fig. 1(Left), and hence, reduce the uncertainty of the gluonic contribution to the proton's spin in a very dramatic way, as shown by the red and yellow bands in Fig. 1(Right). The reduced uncertainties would definitively resolve the question of whether parton spin preferences alone can account for the overall proton spin, or whether an additional contribution 
from the total orbital angular momentum of partons in the nucleon is needed. Clearly, the EIC will make a profound impact on our knowledge of these quantities, unmatched by any other existing or anticipated facility.
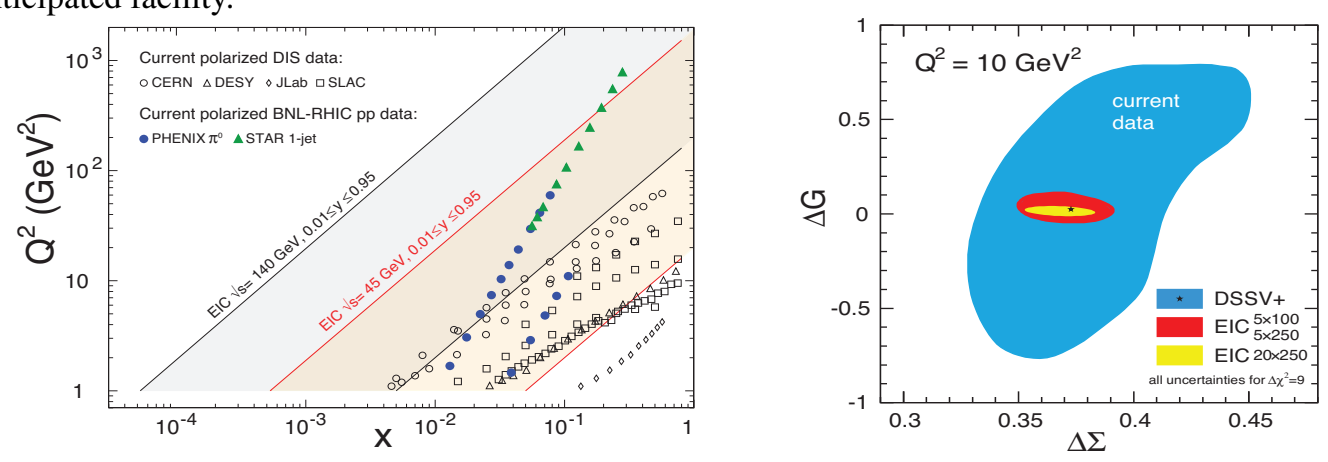

Figure 1. Left: The increase coverage in the proton momentum fraction $x$ vs. the square of the momentum transferred by the electron to the proton $Q^{2}$ in $e+p$ collisions at the EIC. Right: The projected reduction in the uncertainties of gluon and quark helicity contributions to the spin of proton: $\Delta G$ and $\Delta \Sigma$.

\subsection{Confined motion of quarks and gluons inside the nucleon}

Semi-inclusive DIS (SIDIS) measurements have two independent momentum scales: the large momentum transfer from the electron beam that ensures the spatial resolution of the probe, and the momentum of the produced hadrons perpendicular to the direction of the momentum transfer that prefers a small value sensitive to the motion of confined partons. Remarkable theoretical advances over the past decade have led to a rigorous framework where information on the confined motion of the partons inside a fast-moving nucleon is matched to transverse-momentum dependent parton distributions (TMDs). TMDs allow us to investigate the full three-dimensional quark-gluon dynamics inside the proton, about which very little is known to date. In Fig. 2(Left), the probability distribution (the color code) to find the up quarks inside a proton moving in the $z$-direction (out of the page) with its spin polarized in the $y$-direction is plotted as a function of quark transverse-momentum. The anisotropy in transverse momentum is induced by the correlation between the proton's spin direction and the motion of its quarks and gluons. While the figure is based on a preliminary extraction of this distribution from current experimental data, nothing is known about the spin and momentum correlations of the gluons and sea quarks. The EIC will be crucial to initiate and realize such a program, and it will dramatically advance our knowledge of the motion of confined gluons and sea quarks in ways not achievable at any existing or proposed facility.

\subsection{Spatial Imaging of quarks and gluons inside the nucleon}

By choosing particular final states in electron+proton scattering without breaking the proton, the EIC with its unprecedented luminosity and detector coverage will be able to create detailed spatial images of quarks and gluons in the colliding proton. These tomographic images obtained from cross-sections and polarization asymmetries in exclusive processes are encoded in generalized parton distributions (GPDs), and are complementary to those obtained from the TMDs of quarks and gluons, revealing aspects of proton structure that are intimately connected with the dynamics of QCD at large distances. In Fig. 2(Right), the spatial distribution of gluons, as measured in the exclusive process: electron + proton $\rightarrow$ electron + proton $+J / \psi$, is plotted as a function of $b_{T}$, the transverse position of the gluons. No existing or planned facility will be able to perform such measurements. 

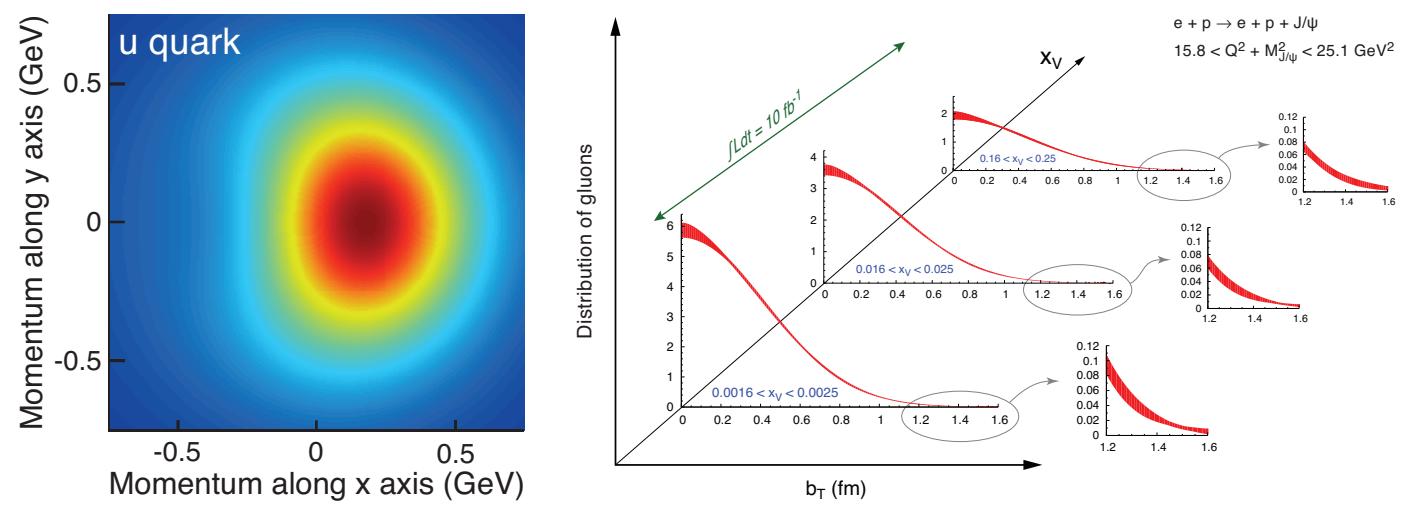

Figure 2. Left: The transverse-momentum distribution of an up quark with longitudinal momentum fraction $x=0.1$ in a transversely polarized proton moving in the $z$-direction, while polarized in the $y$-direction. The color code indicates the probability of finding the up quarks. Right: The projected precision of the transverse spatial distribution of gluons as obtained from the cross-section of exclusive $J / \psi$ production at the EIC.

\section{The nucleus: A femtometer laboratory for QCD}

The emergence of hadrons from almost massless quarks and gluons, or heavy quarks, is a necessary and critical process in the formation of our visible universe shortly after its birth. Color neutralization, is key to this emergent phenomenon of QCD and is likely to take place at the femtometer size, the color confining scale, has yet to be understood within QCD.

\subsection{Quark and gluon landscape in a nucleus}

The EMC experiment at CERN and experiments in the following two decades clearly revealed that the distribution of quarks in a fast-moving nucleus is not a simple superposition of their distributions within nucleons, as shown in Fig. 3. With its much wider kinematic reach in both $x$ and $Q^{2}$, the EIC would allow measurements of the suppression of the structure functions to a much lower value of $x$, approaching the gluon dominated region, where the universal saturation phenomenon related to the gluons in nuclei, is predicted to exist. The EIC could for the first time reliably quantify the probability to find a soft gluon in this interesting regime.

If the nuclear effect in the DIS cross section, as shown in Fig. 3, is mainly due to the abundance of nucleons at the same impact parameter of the nucleus (proportional to $A^{1 / 3}$ ), while the elementary scattering is still relatively weak, one would expect the ratio of nuclear over nucleon structure functions to saturate when $x$ goes below 0.01, as shown for example, by the upper line of the blue area extrapolated from the current data. On the other hand, if the soft gluons are a property of the whole nucleus and the coherence is strong, one would expect the ratio of the nuclear to nucleon structure function to fall continuously as $x$ decreases, as sketched by the lower line of the blue band, and eventually, reach a constant when both nuclear and nucleon structure functions are in the saturation region. From the size of the purple error band in Fig. 3, which is the expected systematic uncertainty at the EIC, it is self-evident that (statistical uncertainty is expected to be much smaller) the EIC will easily distinguish between these two extreme scenarios and light on the nature of soft gluons and sea quarks in a nuclear environment. 

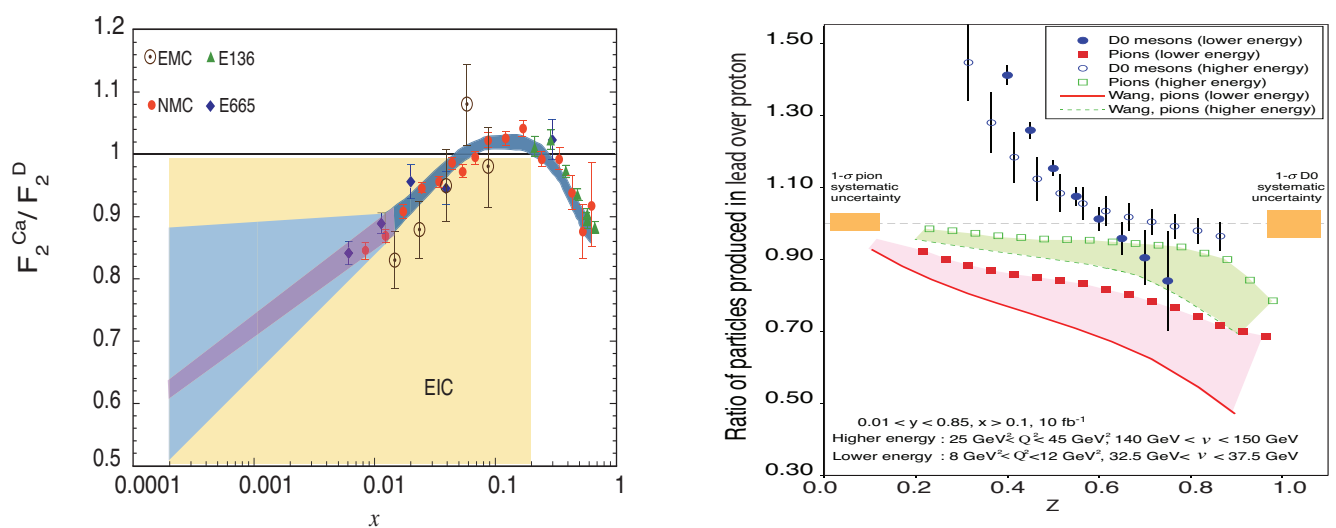

Figure 3. Left: The ratio of nuclear over nucleon $F_{2}$ structure function as a function of Bjorken $x$, with data from existing fixed target DIS experiments at $Q^{2}>1 \mathrm{GeV}^{2}$, along with the QCD global fit from EPS09. Also shown is the expected kinematic coverage of the inclusive measurements at the EIC. The purple error band is the expected systematic uncertainty at the EIC assuming a $\%$ (a total of $4 \%$ ) systematic error, while the statistical uncertainty is expected to be much smaller. Right: The ratio of the semi-inclusive cross-section for producing a pion (red) composed of light quarks, and a $D^{0}$ meson (blue) composed of heavy quarks in $e+$ lead collisions to $e+$ deuteron collisions, plotted as a function of $z$, the ratio of the energy carried by the produced hadron to that of the exchange virtual photon in the rest frame of the colliding proton or ion.

\subsection{The color propagation and neutralization}

The exact mechanism of how colored partons pass through colored media, both cold nuclear matter and the hot quark-gluon plasma (QGP), and their emergence as colorless hadrons, is not understood. At the EIC, a nucleus will provide a femtometer size filter to help determine the correct mechanism by which colored partons interact and hadronize in nuclear matter. By measuring pion and $D^{0}$ meson production in both $e+p$ and $e+A$ collisions, the EIC will provide the first measurement of the quark mass dependence of the response of nuclear matter to a fast moving quark. The dramatic difference between them, shown in Fig. 3 (Right), would be easily discernable at the EIC. The color bands reflect the limitation of our knowledge on hadronization - the emergence of a pion from a colored light quark. Enabling all such studies in one place, the EIC will be a true "femtometer size QCD Laboratory", unique of its kind in the world.

\subsection{QCD matter at an extreme gluon density}

When fast moving hadrons are probed by a high resolution local probe, the low-momentum gluons contained in their wave functions become experimentally accessible. The large soft-gluon density at small- $x$, especially in a large nucleus, enables the gluon-gluon recombination to limit the density growth. Such a self-regulation mechanism necessarily generates a dynamic scale from the interaction of high density massless gluons, known as the saturation scale, $Q_{s}$, at which gluon splitting and recombination reach a balance. At this scale, the density of gluons is expected to saturate, producing new hadronic matter of universal properties. The saturation scale $Q_{s}$ separates the condensed and saturated soft gluonic matter from the dilute, but confined, quarks and gluons in a hadron, as shown in Fig. 4(Left). Such universal cold gluon matter is an emergent phenomenon of QCD dynamics and is of high scientific interest. With its wide kinematic reach, the EIC will be the first experimental 
facility capable of exploring the internal three-dimensional sea quark and gluon structure of a fastmoving nucleus, and the transition from a dilute to a dense gluon density to provide access to a thus far unconfirmed regime of matter where abundant gluons dominate its behavior.
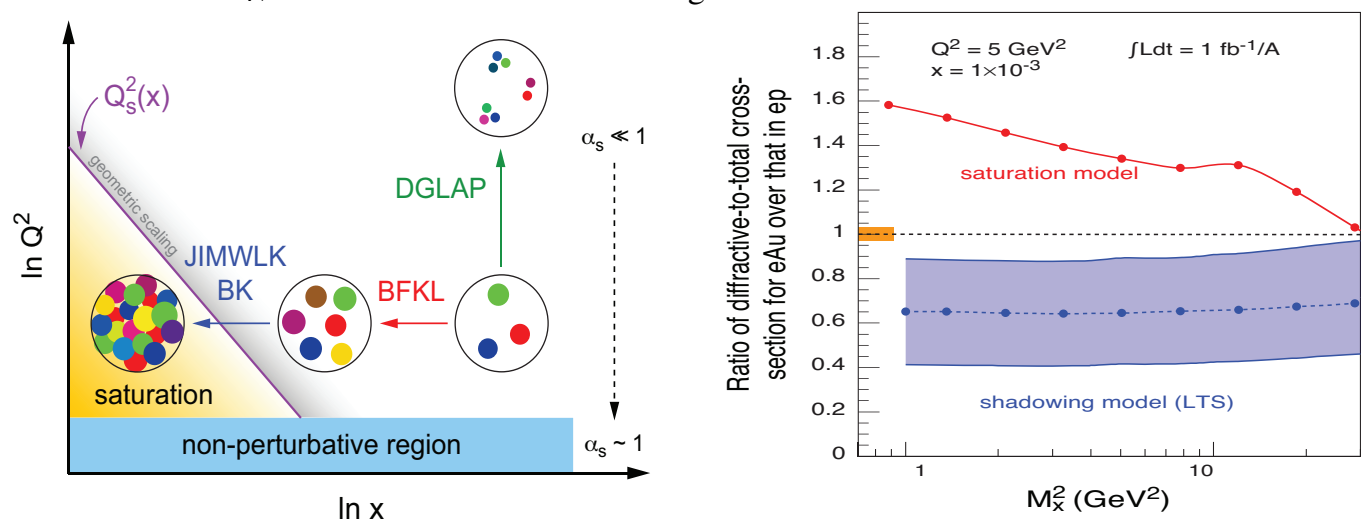

Figure 4. Left: The schematic probe resolution vs. energy landscape, including the regions of low and high saturated parton density, and the transition region between them. Right: The ratio of diffractive over total crosssection for DIS on gold normalized to DIS on proton for different values of mass squared of hadrons produced in the collisions with and without saturation.

The existence of such saturated soft gluon matter is a direct consequence of gluon self-interactions in QCD. With many more soft gluons at the same impact parameters in a large nucleus, enhanced by $A^{1 / 3}$ with the atomic weight $A$ than that in a proton, the gluon saturation is much easier to be reached in $e+A$ than $e+p$ collisions. By measuring the ratio of diffractive cross-sections over the total DIS cross-sections in both $e+A$ and $e+p$ collisions, as shown in Fig. 4(Right), the EIC would provide the first unambiguous evidence for the novel QCD matter of saturated gluons. Furthermore, knowing the properties of this matter and its underlying QCD dynamics is critically important for understanding the dynamical origin of QGP formation from colliding two relativistic heavy ions.

\section{Summary and outlook}

The EIC is a ultimate machine to explore the three-dimensional quark-gluon structure of nucleons and nuclei, to search for hints and clues of the color confinement, to study the color neutralization, to probe the existence of the saturated gluonic matter and to explore it in detail. At extreme energy and luminosities, it may also discover the unexpected phenomena within and beyond the Standard Model. The EIC promises to unite and extend the scientific programs at CEBAF and RHIC in dramatic and fundamentally important ways, and to propel them to the next QCD frontier. Its realization will ensure US leadership in nuclear science research.

Acknowledgements: We thank all members of the EIC White Paper Writing Committee [2] for helpful discussions and the collaboration.

\section{References}

[1] N. Brambilla, S. Eidelman, P. Foka, S. Gardner, A. Kronfeld et al., Eur.Phys.J. C74, 2981 (2014), 1404.3723

[2] A. Accardi, J. Albacete, M. Anselmino, N. Armesto, E. Aschenauer et al. (2012), 1212 . 1701 